\title{
Structure of alkali tellurite glasses from neutron diffraction and molecular orbital calculations
}

\section{$\operatorname{AUTHOR}(S)$ :}

Niida, H; Uchino, T; Jin, JS; Kim, SH; Fukunaga, T; Yoko, $T$

\section{CITATION:}

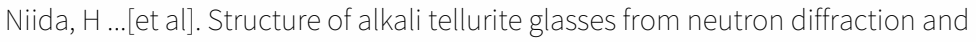
molecular orbital calculations. JOURNAL OF CHEMICAL PHYSICS 2001, 114(1): 459-467

\section{ISSUE DATE:}

2001-01-01

URL:

http://hdl.handle.net/2433/50385

\section{RIGHT:}

Copyright 2001 American Institute of Physics. This article may be downloaded for personal use only. Any other use requires prior permission of the author and the American Institute of Physics. 


\title{
Structure of alkali tellurite glasses from neutron diffraction and molecular orbital calculations
}

\author{
Haruki Niida, Takashi Uchino, ${ }^{\text {a) }}$ and Jisun Jin \\ Institute for Chemical Research, Kyoto University, Uji, Kyoto 611-0011, Japan \\ Sae-Hoon Kim \\ Ceramic Materials Research Institute, Hanyang University, Seoul 133-791, Korea \\ Toshiharu Fukunaga \\ Research Reactor Institute, Kyoto University, Kumatori-cho, Sennan-gun, Osaka 590-0494, Japan \\ Toshinobu Yoko \\ Institute for Chemical Research, Kyoto University, Uji, Kyoto 611-0011, Japan
}

(Received 5 June 2000; accepted 4 October 2000)

\begin{abstract}
The structure of pure $\mathrm{TeO}_{2}$ and alkali tellurite glasses has been examined by neutron diffraction and $a b$ initio molecular orbital methods. The experimental radial distribution functions along with the calculated results have demonstrated that the basic structural units in tellurite glasses change from highly strained $\mathrm{TeO}_{4}$ trigonal bipyramids to more regular $\mathrm{TeO}_{3}$ trigonal pyramids with increasing alkali content. It has also been shown that the $\mathrm{TeO}_{3}$ trigonal pyramids do not exist in the form of isolated units in the glass network but interact with each other to form intertrigonal Te $\cdots \mathrm{O}$ linkages. The present results suggest that nonbridging oxygen (NBO) atoms in tellurite glasses do not exist in their "pure" form; that is, all the $\mathrm{NBO}$ atoms in $\mathrm{TeO}_{3}$ trigonal bipyramids will interact with the first- and/or second-neighbor Te atoms, resulting in the three-dimensional continuous random network even in tellurite glasses with over $30 \mathrm{~mol} \%$ of alkali oxides. (C) 2001 American Institute of Physics. [DOI: 10.1063/1.1328417]
\end{abstract}

\section{INTRODUCTION}

When alkali oxides $\left(\mathrm{R}_{2} \mathrm{O}\right)$ are added into $\mathrm{SiO}_{2}$ glass, the $\mathrm{Si}-\mathrm{O}-\mathrm{Si}$ network is broken to form the so-called "nonbridging oxygens (NBOs)" without changing the coordination number, $N$, of oxygen around the silicon atoms $(N=4)$. However, addition of $\mathrm{R}_{2} \mathrm{O}$ into tellurium oxide based glasses, which have attracted much interest because of their high refractive index, wide infrared transmittance, and large thirdorder nonlinear optical susceptibility, ${ }^{1-5}$ results in much more complicated structural changes as compared with those for silicate glasses. As $\mathrm{R}_{2} \mathrm{O}$ is added into $\mathrm{TeO}_{2}$ glass, which comprises the $\mathrm{TeO}_{4}$ trigonal bipyramids (tbps), it has been proposed that the basic structural unit in the glasses changes from $\mathrm{TeO}_{4}$ to $\mathrm{TeO}_{3+1}$ and/or $\mathrm{TeO}_{3}$ trigonal pyramids (tps). ${ }^{6-8}$ Accordingly, the tellurite glasses are believed to consist of isolated structural units such as $\mathrm{Te}_{2} \mathrm{O}_{5}^{2-}$ and $\mathrm{TeO}_{3}^{2-}$ having NBO atoms. ${ }^{6,9,10}$ It has also been suggested that the structure of alkali tellurite glasses degrades with increasing temperature, accompanied by the conversion of $\mathrm{TeO}_{4}$ tbps into $\mathrm{TeO}_{3}$ tps as well. ${ }^{7,11}$

It is most likely that the NBO atoms in tellurite and silicate glasses will behave as charge compensators of positively charged alkali cations incorporated into the glass structure. It is hence reasonable to expect that the electronic structure of NBOs is basically different from that of bridging oxygens (BOs). Indeed, as for alkali silicate glasses, such a difference in the electronic structure between $\mathrm{BO}$ and NBO

${ }^{a)}$ Author to whom correspondence should be addressed. atoms can be observed as a chemical shift in the X-ray photoelectron spectroscopy (XPS); ${ }^{12,13}$ the $\mathrm{O} 1 s$ photoelectron energies for the $\mathrm{BO}$ atoms in silicate glasses are generally higher than those for the NBO atoms by $\sim 2 \mathrm{eV}$. Recently, Himei et al. ${ }^{14}$ however, have shown that the O 1 s XPS spectra of alkali tellurite glasses do not show such two distinct peaks that are attributed to $\mathrm{BO}$ and $\mathrm{NBO}$ atoms but exhibit only a single peak; the full widths at half maximum (FWHM) of the observed O $1 s$ peak almost remain constant even when modifiers are added into $\mathrm{TeO}_{2}$ glass. These experimental results suggest that as far as the $\mathrm{O} 1 s$ photoelectron energies are concerned, one cannot distinguish between $\mathrm{BO}$ and NBO atoms. That is, all the oxygen atoms in alkali tellurite glasses may have the same electronic structure.

The above experimental results certainly manifest a singular nature of the NBOs in alkali tellurite glasses. In actual tellurite glasses, structural fragments such as $\mathrm{Te}_{2} \mathrm{O}_{5}^{2-}$ and $\mathrm{TeO}_{3}^{2-}$ units presumably interact with each other, and, accordingly, the electronic structure and charge distributions of these structural units will be affected as a result of these interactions. Unfortunately, however, complete knowledge about the structure of the glass network in tellurite glasses is still lacking and, therefore, a satisfactory interpretation of these XPS spectra ${ }^{14}$ has not yet been given.

In this paper, we hence investigate the structure of alkali tellurite glasses by neutron diffraction and $a b$ initio molecular orbital (MO) calculations on clusters of atoms modeling the local structures of sodium tellurite as well as pure $\mathrm{TeO}_{2}$ glasses. The radial distribution functions obtained from the 
neutron diffraction measurements will give information about the first-coordination shells of Te, namely, the shortrange structure of the tellurite glasses. On the other hand, cluster calculations will be useful to get a better knowledge about the electronic structure of the glasses. We have previously calculated $\mathrm{O} 1 \mathrm{~s}$ photoelectron energies of sodium silicate $^{15}$ and sodium aluminosilicate ${ }^{16}$ glasses by using appropriate cluster models. The calculations have successfully reproduced the observed chemical shift between $\mathrm{BO}$ and NBO atoms, indicating that the core binding energies are determined mostly by the charge distribution of the first- and second-coordination environments of an atom of interest. Thus, the present cluster calculations along with the neutron diffraction measurements will provide a new insight into the unsolved problems concerning the glass network and the electronic structure of alkali tellurite glasses.

On the basis of the experimental and calculated results, we discuss how the proposed structural fragments interact to form a continuous random network and provide a possible model about the short- and intermediate-range structures of alkali tellurite glasses.

\section{EXPERIMENTAL AND CALCULATIONAL PROCEDURES}

\section{A. Preparation of samples}

Glasses of compositions $x \mathrm{Li}_{2} \mathrm{O} \cdot(100-x) \mathrm{TeO}_{2} \quad(x=0$, $10,20,30)$ and $x \mathrm{Na}_{2} \mathrm{O} \cdot(100-x) \mathrm{TeO}_{2}(x=0,10,20,30,33$, 37) were prepared from reagent grade $\mathrm{Li}_{2} \mathrm{CO}_{3}, \mathrm{Na}_{2} \mathrm{CO}_{3}$, and crystalline $\beta-\mathrm{TeO}_{2}$ powder by melt quenching. About $10 \mathrm{~g}$ batches were melted in a Pt-5\% Au crucible at $750-800{ }^{\circ} \mathrm{C}$ for 10-20 min. The melts were rapidly quenched down to $-11^{\circ} \mathrm{C}$ by dropping the bottom of the crucible into a freezing mixture consisting of ice, ethanol, and $\mathrm{NaCl}$, resulting in transparent alkali tellurite glasses, which were shown to be amorphous by $\mathrm{x}$-ray powder diffraction.

\section{B. Neutron diffraction}

The neutron diffraction experiments were performed on a high intensity total scattering spectrometer (HIT-II) with the time-of-flight pulsed neutron source at the High Energy Accelerator Research Organization in Tsukuba, Japan. The glass samples were placed in thin-walled $(25 \mu \mathrm{m})$ vanadium cell with a diameter of $8 \mathrm{~mm}$. The experiments were carried out under vacuum at room temperature. Time-of-flight spectra were recorded separately for each group of detectors at nominal scattering angles of $150^{\circ}, 90^{\circ}, 50^{\circ}, 30^{\circ}, 23^{\circ}, 13^{\circ}$, and $8^{\circ}$. The data were corrected for subtraction of cell intensity, absorption, multiple scattering, and normalization with vanadium standard to obtain the structure factor, $S(Q)$, following the procedure described in Ref. 17. The structure factor of each sample was truncated at $Q_{\max }=28 \AA^{-1}$, and the radial distribution function, $\boldsymbol{J}(\boldsymbol{r})$, was obtained by a Fourier transformation,

$$
\boldsymbol{J}(\boldsymbol{r})=4 \pi \boldsymbol{r}^{2} \rho_{0}+\frac{2 \boldsymbol{r}}{\pi} \int_{0}^{Q_{\max }} \boldsymbol{Q}[S(\boldsymbol{Q})-1] \sin (\boldsymbol{Q} \boldsymbol{r}) d \boldsymbol{Q},
$$

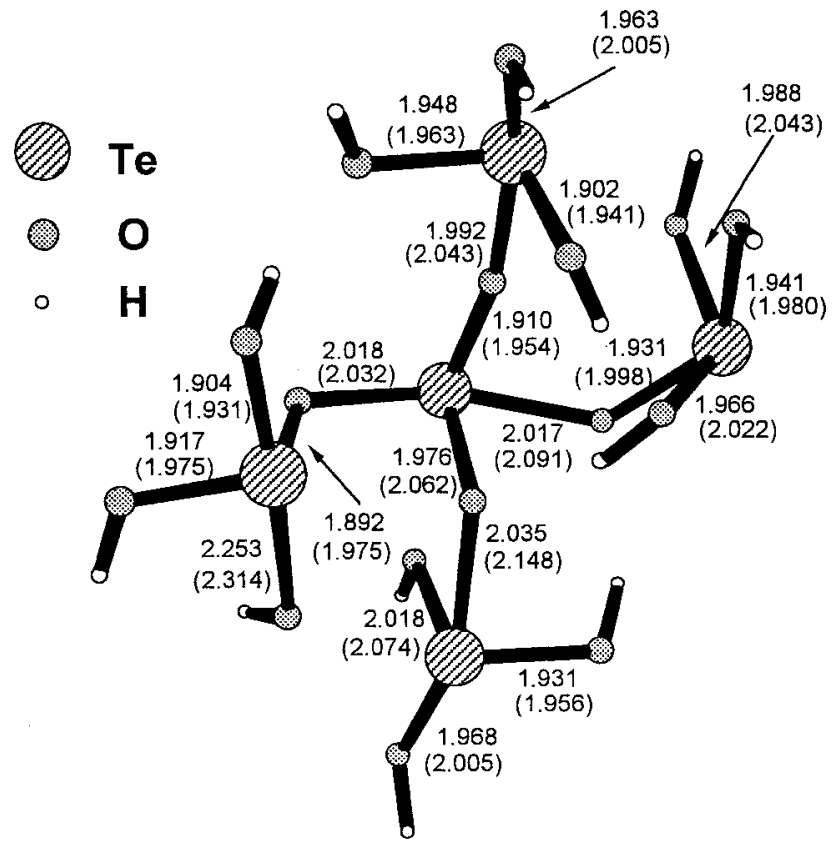

average $\mathrm{Te}-\mathrm{O}=1.974 \mathrm{~A}$

(2.028)

FIG. 1. Optimized structure of the $\mathrm{Te}_{5} \mathrm{O}_{16} \mathrm{H}_{12}$ cluster (model 1). Principal $\mathrm{HF} / 3-21 \mathrm{G}(*)$ and $\mathrm{B} 3 \mathrm{LYP} / 3-21 \mathrm{G}(*)$ (values in parentheses) bond distances are shown in $\AA$.

where $\rho_{0}$ is the average number density. Furthermore, we introduced a Lorch modification function ${ }^{18}$ in the integrand in Eq. (1) to reduce spurious truncation ripples in the radical distribution function. In what follows, we will refer to the radical distribution function modified by the Lorch function as $\boldsymbol{J}^{\bmod }(\boldsymbol{r})$.

\section{Models for $a b$ initio molecular orbital calculations}

Previously, we have carried out ab initio cluster model calculations to investigate the vibrational properties of pure $\mathrm{TeO}_{2}$ glass. ${ }^{19}$ In a previous paper, we used the model clusters consisting of one $\mathrm{TeO}_{4}$ or two $\mathrm{TeO}_{4}$ tbp(s) and have shown that the basic vibrational properties of $\mathrm{TeO}_{2}$ glass are reasonably reproduced by this method. In this work, we use a larger cluster having five $\mathrm{TeO}_{4}$ tbps (model 1, see Fig. 1) to model the structure of $\mathrm{TeO}_{2}$ glass on the medium-range as well as short-range length scales. The "surface" oxygen atoms in the cluster were terminated by hydrogen atoms, and its geometry was fully optimized at the Hartree-Fock (HF) and density functional theory (DFT) levels using the 3-21G basis set augmented by $d$ functions on the Te (and $\mathrm{Na}$ ) atom(s), ${ }^{20}$ which is referred to as $3-21 \mathrm{G}(*)$. A value of 0.237 was employed as the exponent for the $d$ functions of Te. ${ }^{21}$ Becke's three-parameter hybrid method ${ }^{22}$ using the LeeYang-Parr correlation functional ${ }^{23}$ (B3LYP) was employed in all DFT calculations.

As a model of alkali tellurite glasses, we first employ the $\mathrm{Te}_{2} \mathrm{O}_{5}^{2-}\left(\mathrm{Na}^{+}\right)_{2}$ cluster (see Fig. 2), which can be regarded as one of the major constituent isolated structural units in sodium tellurite glasses having more than $30 \mathrm{~mol} \% \mathrm{Na}_{2} \mathrm{O}$. This model consists of two $\mathrm{TeO}_{3}$ unit that shares one bridg- 


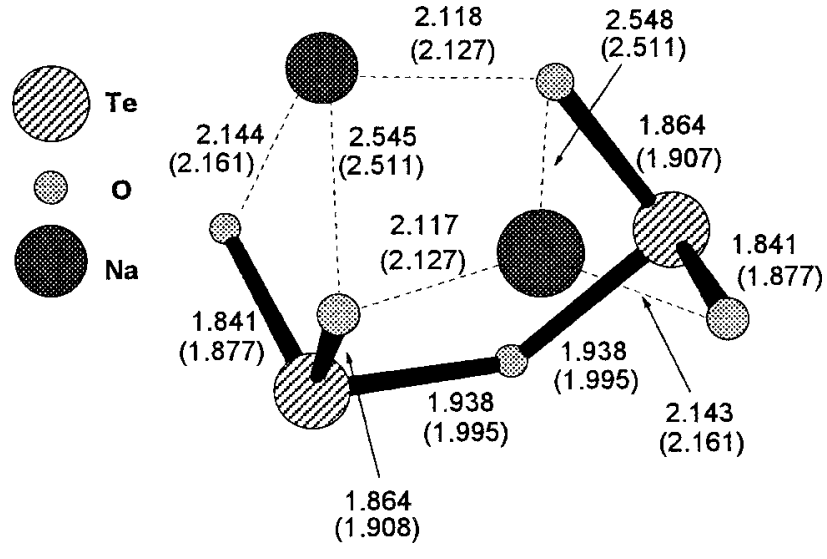

FIG. 2. Optimized structure of the $\mathrm{Te}_{2} \mathrm{O}_{5} \mathrm{Na}_{2}$ cluster (model 2). Principal $\mathrm{HF} / 3-21 \mathrm{G}\left({ }^{*}\right)$ and $\mathrm{B} 3 \mathrm{LYP} / 3-21 \mathrm{G}\left({ }^{*}\right)$ (values in parentheses) bond distances are shown in $\AA$.

ing oxygen; each $\mathrm{TeO}_{3}$ unit has two terminal $\mathrm{Te}-\mathrm{O}$ bonds. In what follows, we refer to this model as model 2 . In order to evaluate the size effect on the structure of the model clusters, we next employ a larger cluster consisting of three $\mathrm{Te}_{2} \mathrm{O}_{5}^{2-}\left(\mathrm{Na}^{+}\right)_{2}$ structural units (model 3, see Fig. 3). The geometries of the above sodium tellurite clusters were optimized at the HF/3-21G(*) and B3LYP/3-21G(*) levels without imposing any structural constraints. As for model 3, several minimum energy structures are possible to exist depending the initial configuration used for geometry optimization; however, we report here only one optimal structure since the geometry optimization of such a large cluster requires a large amount of CPU time. The $\mathrm{O} 1 s$ photoelectron energies of the clusters were calculated on the basis of the Koopmans' theorem, ${ }^{24}$ which equates the photoelectron energy to the negative value of the one-electron energy of the corresponding orbital calculated at the HF level. As we ex- pect in view of our use of Koopmans' theorem, which neglects the electronic reorganization energy, there may be a considerable discrepancy between calculated and experimental energies. However, it has been recognized that for atoms in the same molecule the relative energy shifts of the core levels can be estimated reliably by the this theorem. ${ }^{25}$

All ab initio MO calculations in this study were performed using the GAUSSIAN 94 program $^{26}$ on a supercomputer CRAY T94/4128.

\section{RESULTS}

\section{A. Neutron diffraction}

The structure factors, $S(Q)$, of the $x \mathrm{Li}_{2} \mathrm{O} \cdot(100$ $-x) \mathrm{TeO}_{2}(x=0,10,20,30)$ and $x \mathrm{Na}_{2} \mathrm{O} \cdot(100-x) \mathrm{TeO}_{2}$ $(x=0,10,20,30,33,37)$ are shown in Fig. 4 . We see from Fig. 4 that all the samples show similar oscillatory behaviors for the high- $Q$ range, indicating very similar short-range structures within the glass network. Figures 5 and 6 show the radial distribution functions of the lithium and sodium tellurite glasses, respectively.

It is clear from Figs. 5 and 6 that the Lorch modification function considerably reduces truncation ripples in the respective radical distribution function, but this is accomplished at the expense of a real-space resolution. The main differences between $\boldsymbol{J}(\boldsymbol{r})$ and $\boldsymbol{J}^{\mathrm{mod}}(\boldsymbol{r})$ can be seen in the distance range from $\sim 2.0$ to $\sim 2.4 \AA$. In $\boldsymbol{J}(\boldsymbol{r})$ of lithium tellurite glasses [see Fig. 5(a)], for example, we see several peaks in this distance range, whereas in $\boldsymbol{J}^{\bmod }(\boldsymbol{r})$ [see Fig. 5(b)] such peaks are smeared as a result of the introduction of the Lorch modification function. It should be noted, however, that $\boldsymbol{J}^{\mathrm{mod}}(\boldsymbol{r})$ does show the atomic correlations on the longer distance side of the main peak at $\sim 1.9 \AA$ for all the glass samples investigated. We, therefore, consider that the observed peaks in the range from $\sim 2.0$ to $\sim 2.4 \AA$ that can be

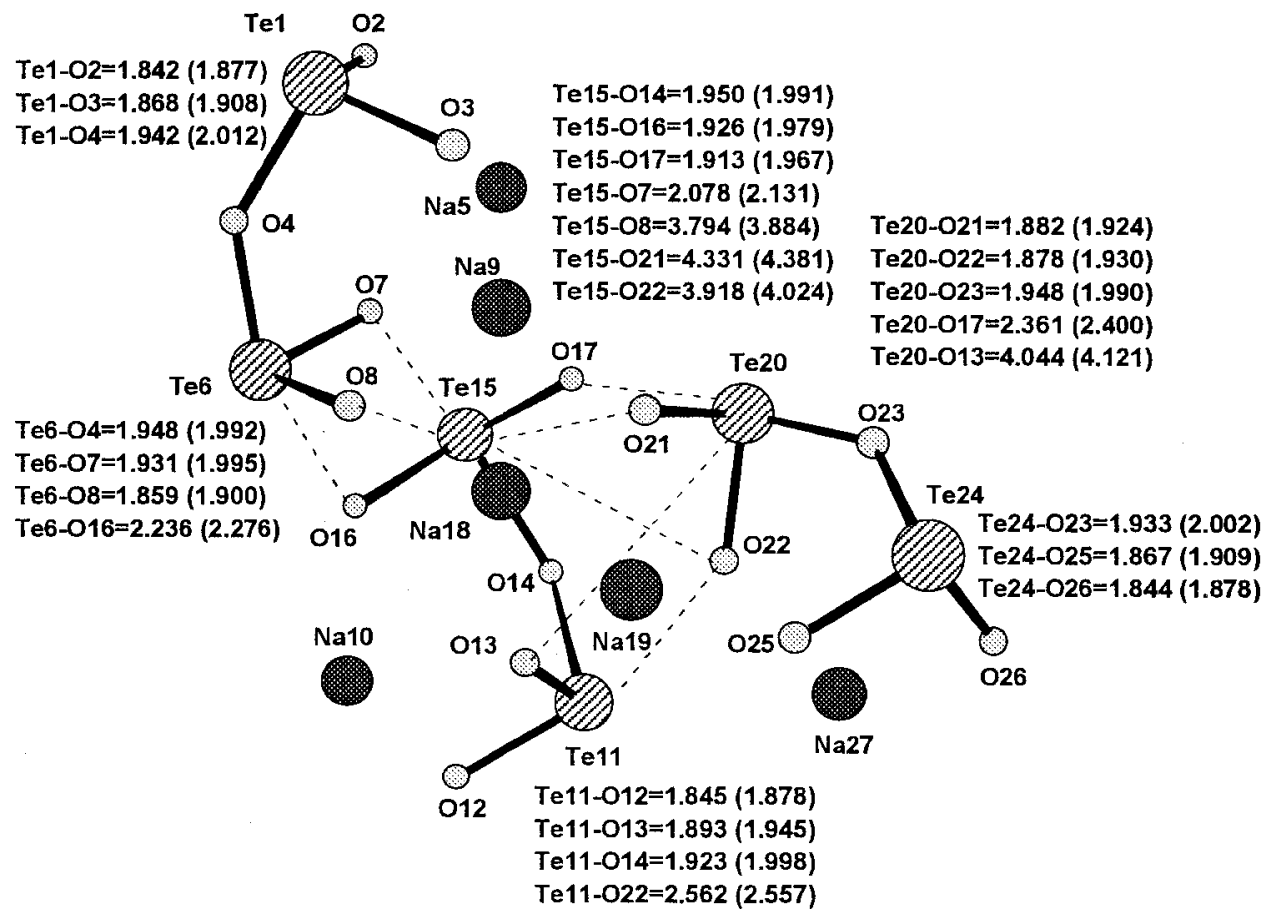

FIG. 3. Optimized structure of the $3\left(\mathrm{Te}_{2} \mathrm{O}_{5} \mathrm{Na}_{2}\right)$ cluster (model 3). Principal $\mathrm{HF} / 3-21 \mathrm{G}\left({ }^{*}\right)$ and B3LYP/ 3-21G(*) (values in parentheses) bond distances are shown in $\AA$. Broken lines indicate the intertrigonal $\mathrm{Te}-\mathrm{O}$ correlations in the distance range below $4.5 \AA$ A. 

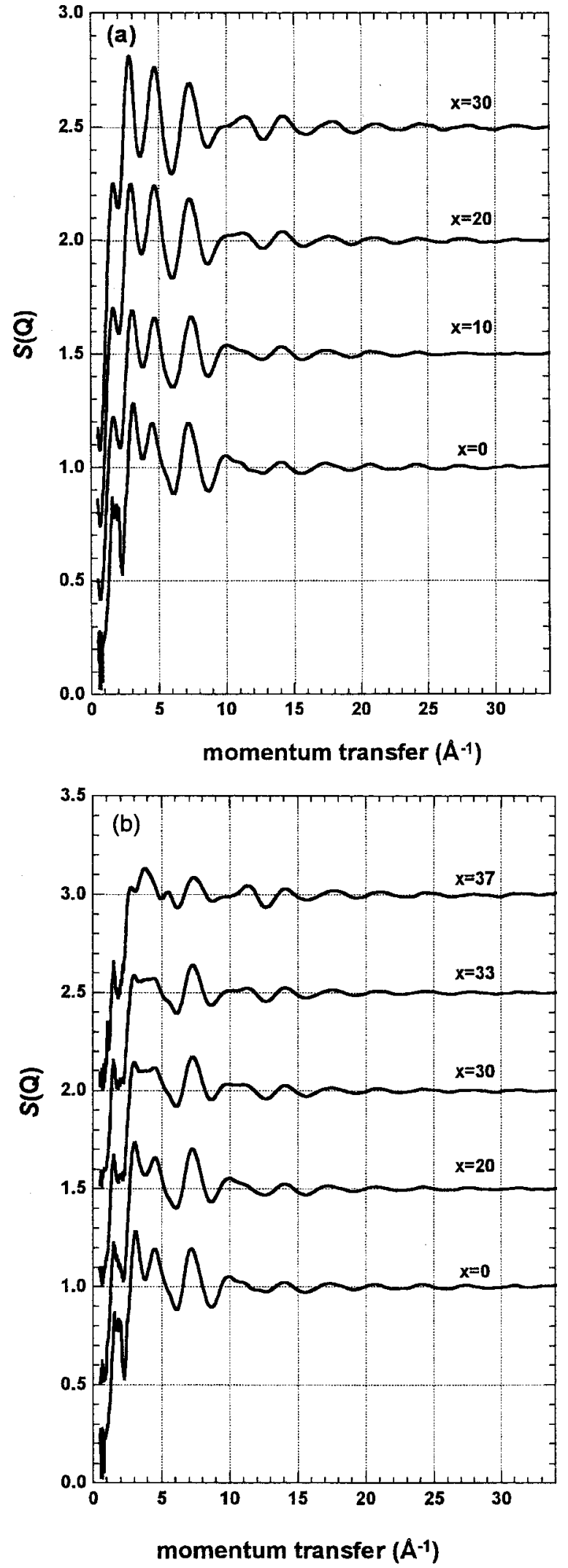

FIG. 4. Experimental neutron structure factors $S(Q)$ of (a) $x \mathrm{Li}_{2} \mathrm{O} \cdot(100$ $-x) \mathrm{TeO}_{2}(x=0,10,20,30)$ and (b) $x \mathrm{Na}_{2} \mathrm{O} \cdot(100-x) \mathrm{TeO}_{2}(x=0,10,20,30$, $33,37)$ glasses. Consecutive curves are shifted vertically by 0.5 for clarity.

seen in $\boldsymbol{J}(\boldsymbol{r})$ of the present glass samples are not artifacts although their peak positions may be affected by possible truncation ripples. The coordination numbers shown below
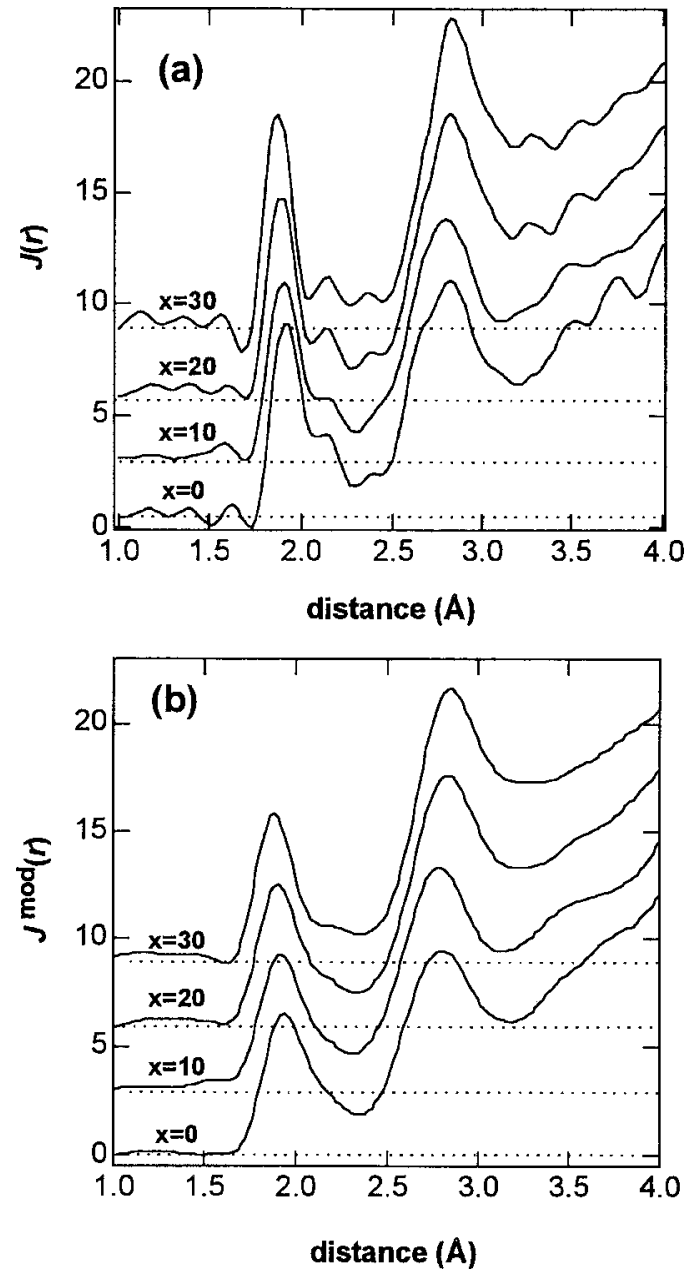

FIG. 5. Experimental radial distribution functions of $x \mathrm{Li}_{2} \mathrm{O} \cdot(100$ $-x) \mathrm{TeO}_{2}(x=0,10,20,30)$ glasses obtained (a) without and (b) with using the Lorch modification function. Consecutive curves are shifted vertically by 3.0 for clarity.

are hence calculated on the basis of the $\boldsymbol{J}(\boldsymbol{r})$ that is not modified by the Lorch function.

As shown in Fig. 7(a), the $\boldsymbol{J}(\boldsymbol{r})$ of pure $\mathrm{TeO}_{2}$ glass exhibits three Te-O correlations for $r \$ 2.4 \AA$. It is worth mentioning that the broad $\mathrm{Te}-\mathrm{O}$ peak in $\boldsymbol{J}^{\mathrm{mod}}(\boldsymbol{r})$ of pure $\mathrm{TeO}_{2}$ glass can also be deconvoluted into three $\mathrm{Te}-\mathrm{O}$ peaks as shown in Fig. 7(b), confirming that the $\mathrm{Te}-\mathrm{O}$ peaks in the distance range from $\sim 2.0$ to $\sim 2.4 \AA$ are not due to the truncation ripples. The total coordination number obtained for these $\mathrm{Te}-\mathrm{O}$ correlations is calculated to be $\sim 4$ (see Table I). This result indicates that the $\mathrm{Te}-\mathrm{O}$ correlations in the range from $\sim 2.0$ to $\sim 2.4 \AA$ correspond to the first coordination shell of the four-coordinated $\mathrm{Te}$, namely, $\mathrm{TeO}_{4}$ tbp units, although these structural units are expected to be highly deformed in the corresponding glass network.

It is interesting to note that the longer pair correlations for $\sim 2.0<r \lesssim 2.4 \AA$ along with the main $\mathrm{Te}-\mathrm{O}$ peak at $\sim 1.9 \AA$ still remain even when alkali cations are introduced into the $\mathrm{TeO}_{2}$ structure irrespective of the type of alkali (see Figs. 5 and 6). Since natural lithium has a negative scattering length, correlations between $\mathrm{Li}$ and a nucleus having a positive scattering length (e.g., $\mathrm{O}$ and $\mathrm{Te}$ ) result in negative peaks in $\boldsymbol{J}(\boldsymbol{r})$. For example, the nearest $\mathrm{Li}-\mathrm{O}$ correlations 

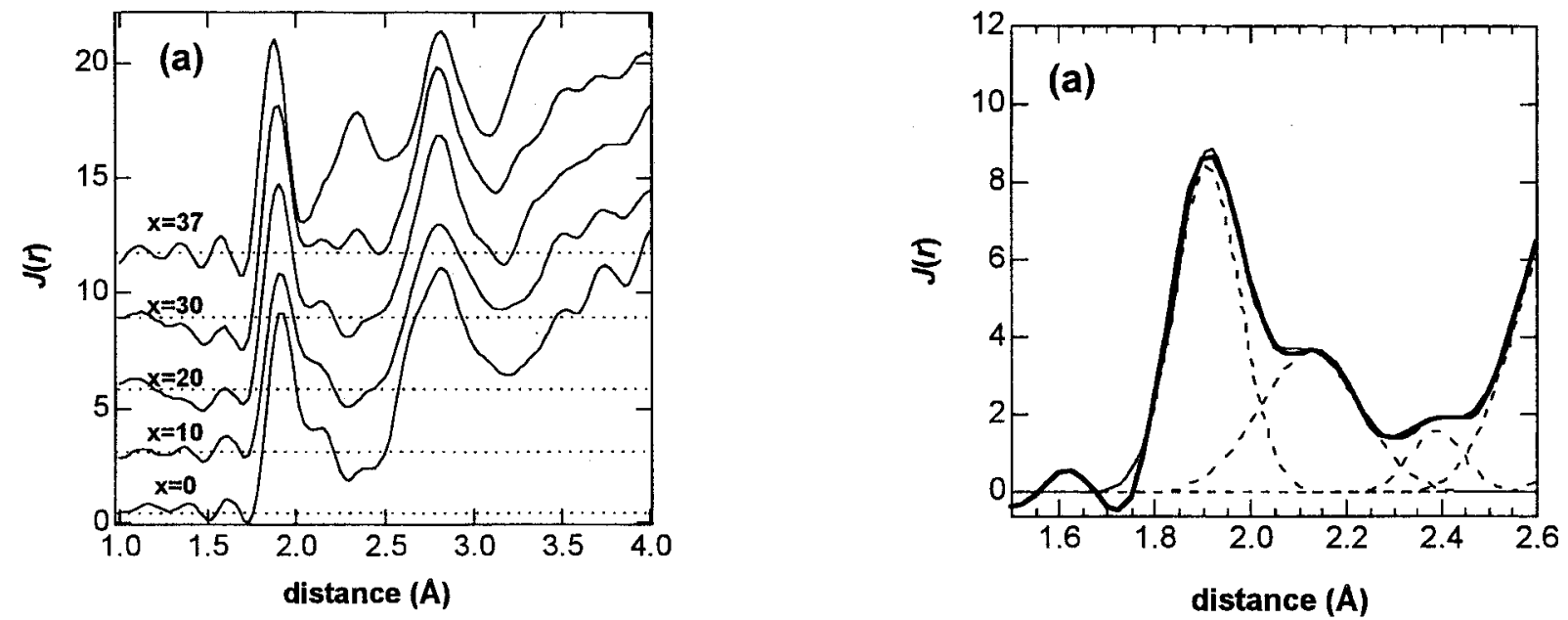

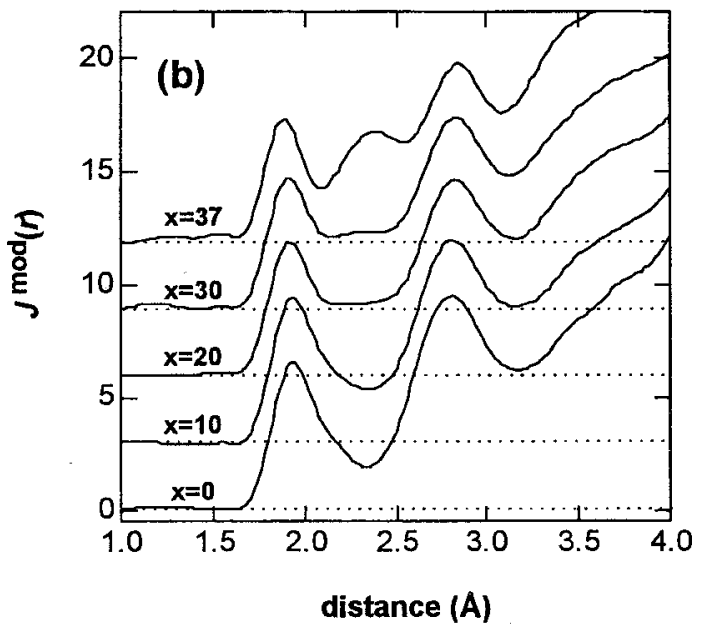

FIG. 6. Experimental radial distribution functions of $x \mathrm{Na}_{2} \mathrm{O} \cdot(100$ $-x) \mathrm{TeO}_{2}(x=0,10,20,30,37)$ glasses obtained (a) without and (b) with using the Lorch modification function. Consecutive curves are shifted vertically by 3.0 for clarity.

will cause a negative peak around $2 \AA$. However, we do not see such a negative region in $\boldsymbol{J}(\boldsymbol{r})$ of lithium tellurite glasses. On the contrary, as mentioned above, there certainly exist positive correlations in the distance range form $\sim 2.0$ to $\sim 2.4$ $\AA$, indicating that these positive contributions in $\boldsymbol{J}(\boldsymbol{r})$ of lithium tellurite glasses are most likely responsible for the $\mathrm{Te}-\mathrm{O}$ correlations that overwhelm the negative $\mathrm{Li}-\mathrm{O}$ contributions. That is, the coordination environment of $\mathrm{Te}$ in lithium tellurite glasses will be characterized not only by a single $\mathrm{Te}-\mathrm{O}$ distance at $\sim 1.9 \AA$ but also by longer $(r$ $\geq 2.0 \AA$ ) $\mathrm{Te}-\mathrm{O}$ correlations. On the other hand, the nearest $\mathrm{Na}-\mathrm{O}$ correlation will yield a positive peak around $2.3 \AA$. A relatively large peak at $\sim 2.3 \AA$ seen in $J(r)$ of $37 \mathrm{Na}_{2} \mathrm{O} \cdot 63 \mathrm{TeO}_{2}$ glass is hence attributed to the nearest $\mathrm{Na}-\mathrm{O}$ as well as longer $\mathrm{Te}-\mathrm{O}$ correlations.

In Table I, we show the coordination number, $N$, of the first $\mathrm{Te}-\mathrm{O}$ peak at $\sim 1.9 \AA$ in $J(r)$ of sodium tellurite glasses. Since the coordination numbers of the other $\mathrm{Te}-\mathrm{O}$ peaks in alkali tellurite glasses are expected to include possible errors derived from the $\mathrm{Li}-\mathrm{O}$ and $\mathrm{Na}-\mathrm{O}$ correlations, we did not obtain their coordination numbers. We notice from Table I and Figs. 5 and 6 that the first Te-O peak becomes narrow with $\mathrm{Li}$ or $\mathrm{Na}$ addition and its coordination

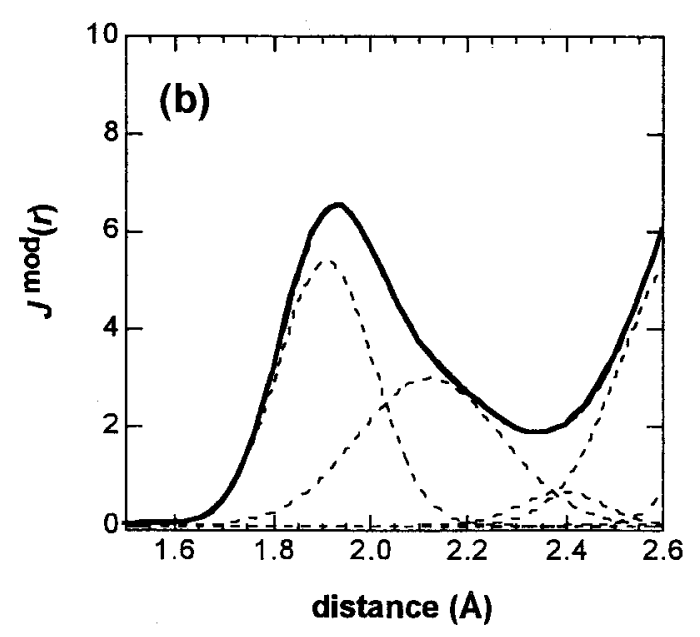

FIG. 7. Deconvoluted Gaussian profiles (broken lines) of the Te-O peaks in (a) $\boldsymbol{J}(\boldsymbol{r})$ and (b) $\boldsymbol{J}^{\mathrm{mod}}(\boldsymbol{r})$ of pure $\mathrm{TeO}_{2}$ glass. The experimental curves are shown in thick solid lines, and the model functions from the least-squares fits are shown in thin solid lines. As for $\boldsymbol{J}^{\text {mod }}(\boldsymbol{r})$, we used the fixed peak positions obtained from the fitting of the Te-O peaks in $\boldsymbol{J}(\boldsymbol{r})$ of pure $\mathrm{TeO}_{2}$ glass.

number increases up to $\sim 3$. This implies that the basic structural units in tellurite glasses change from highly deformed $\mathrm{TeO}_{4}$ to rather regular $\mathrm{TeO}_{3}$ units with increasing alkali content. Also, the first Te-O peak at $\sim 1.9 \AA$ in $\boldsymbol{J}(\boldsymbol{r})$ obtained for sodium tellurite glasses [see Fig. 6(a)] having over $30 \mathrm{~mol} \%$ of $\mathrm{Na}_{2} \mathrm{O}$ cannot be further deconvoluted into two separate

TABLE I. Peak positions (in $\AA$ ) of the first, second, and third Te-O distances obtained from the $\boldsymbol{J}(\boldsymbol{r})$ shown in Figs. 5(a) and 6(a). Values in parentheses show the coordination numbers for the corresponding peaks.

\begin{tabular}{clll}
\hline \hline Glass composition & First Te-O & Second Te-O & Third Te-O \\
\hline $\mathrm{TeO}_{2}$ & $1.93(2.2)$ & $2.13(1.5)$ & $2.39(0.4)$ \\
$10 \mathrm{Li}_{2} \mathrm{O} \cdot 90 \mathrm{TeO}_{2}$ & 1.90 & 2.13 & 2.40 \\
$20 \mathrm{Li}_{2} \mathrm{O} \cdot 80 \mathrm{TeO}_{2}$ & 1.90 & 2.13 & 2.38 \\
$30 \mathrm{Li}_{2} \mathrm{O} \cdot 70 \mathrm{TeO}_{2}$ & 1.88 & 2.15 & 2.38 \\
$10 \mathrm{Na}_{2} \mathrm{O} \cdot 90 \mathrm{TeO}_{2}$ & $1.90(2.2)$ & 2.12 & $2.40^{\mathrm{a}}$ \\
$20 \mathrm{Na}_{2} \mathrm{O} \cdot 80 \mathrm{TeO}_{2}$ & $1.90(2.8)$ & 2.14 & $2.40^{\mathrm{a}}$ \\
$30 \mathrm{Na}_{2} \mathrm{O} \cdot 70 \mathrm{TeO}_{2}$ & $1.90(3.0)$ & 2.14 & $2.33^{\mathrm{a}}$ \\
$37 \mathrm{Na}_{2} \mathrm{O} \cdot 63 \mathrm{TeO}_{2}$ & $1.87(3.1)$ & 2.15 & $2.32^{\mathrm{a}}$ \\
\hline \hline
\end{tabular}

${ }^{a}$ The peak positions of the third $\mathrm{Te}-\mathrm{O}$ correlation in sodium tellurite glasses may be effected by the $\mathrm{Na}-\mathrm{O}$ correlations at $\sim 2.4 \AA$. 
peaks. As for sodium silicate glasses, the $\mathrm{Si}-\mathrm{O}$ peak in the neutron radial distribution functions can be separated into two peaks due to the $\mathrm{Si}-\mathrm{BO}(\sim 1.64 \AA)$ and $\mathrm{Si}-\mathrm{NBO}(\sim 1.59$ $\AA$ ) bonds. ${ }^{27}$ It is hence probable that three $\mathrm{Te}-\mathrm{O}$ bonds in the $\mathrm{TeO}_{3}$ units yield similar bond distances and that the $\mathrm{Te}-\mathrm{BO}$ and $\mathrm{Te}-\mathrm{NBO}$ bonds may not be clearly differentiated in terms of their bond distances.

It should be worth mentioning that in $\boldsymbol{J}(\boldsymbol{r})$ of lithium tellurite glasses one sees longer $\mathrm{Te}-\mathrm{O}$ correlations at $\sim 2.0$ to $\sim 2.4 \AA$ in addition to the main $1.9 \AA$ peak as pointed out previously. This result most likely suggests that the $\mathrm{TeO}_{3}$ tbps in alkali tellurite glasses interact with each other, yielding these longer intertrigonal $\mathrm{Te} \cdots \mathrm{O}$ correlations in the first coordination shell.

\section{B. Molecular orbital calculations}

The HF/3-21G(*) and B3LYP/3-21G(*) geometries of the cluster modeling the local structure of $\mathrm{TeO}_{2}$ glass (model 1) are depicted in Fig. 1. The attainment of the energy minimum was verified because no imaginary frequencies were obtained for these clusters. It has been found that although the $\mathrm{Te}-\mathrm{O}$ bond distances calculated at the $\mathrm{B} 3 \mathrm{LYP} / 3-21 \mathrm{G}(*)$ level tend to become longer than those at the HF/3-21G(*) level, the resultant optimized geometries are almost identical irrespective of the calculated levels of theory used. As will be shown in Figs. 2 and 3, this tendency is also true for models 2 and 3. Figure 8 shows the distribution of the Te-O bond distances in the constituent five $\mathrm{TeO}_{4}$ units calculated at the $\mathrm{HF} / 3-21 \mathrm{G}(*)$ level for model 1. One notices from Fig. 8 that the calculated $\mathrm{Te}-\mathrm{O}$ bond distances range from $\sim 1.9$ to $\sim 2.3 \AA$. Such a broad distribution of the $\mathrm{Te}-\mathrm{O}$ bond distances is in agreement with the observed broad $\mathrm{Te}-\mathrm{O}$ correlations of $\mathrm{TeO}_{2}$ glass shown in Figs. 5, 6, and 7. Thus obtained tendency is in contrast with rather a narrow distribution of the $\mathrm{Si}-\mathrm{O}$ bond distances calculated for the $\mathrm{SiO}_{4}$ tetrahedral units in a silica cluster reported previously ${ }^{28}$ (see also Fig. 8). These calculated and observed results allow us to conclude that the respective $\mathrm{TeO}_{4}$ units in $\mathrm{TeO}_{2}$ glass are intrinsically highly deformed from the ideal trigonal bipyramidal structures.

We next turn to the results of model 2 shown in Fig. 2. From Fig. 2, one sees that each $\mathrm{TeO}_{3}$ unit has a trigonal pyramid structure and that there is no apparent distinction between the two Te-NBO bond distances in each $\mathrm{TeO}_{3}$ unit. This result indicates that a resonance occurs between these two $\mathrm{Te}-\mathrm{O}$ bonds via $\mathrm{Na}-\mathrm{NBO}$ interactions. Furthermore, one should note that each $\mathrm{Na}$ atom in model 2 interacts not only with two NBOs in one $\mathrm{TeO}_{3}$ unit but also with another $\mathrm{NBO}$ in the adjacent $\mathrm{TeO}_{3}$ unit, showing a trifurcate coordination of $\mathrm{Na}$. This result suggests that further resonance among three or more NBOs occurs in actual tellurite glasses. In model 2, however, the $\mathrm{Te}-\mathrm{BO}$ bond distances are appreciably longer than the Te-NBO bond distances by $\sim 0.09 \AA$, which may not be consistent with the observed single $\mathrm{Te}-\mathrm{O}$ peak at $\sim 1.9 \AA$ in $\boldsymbol{J}(r)$ mentioned above.

The optimized geometry of model 3 , which is composed of three $\mathrm{Te}_{2} \mathrm{O}_{5}^{2-}\left(\mathrm{Na}^{+}\right)_{2}$ units, is shown in Fig. 3. We confirmed that the optimized geometry of model 3 as well as that
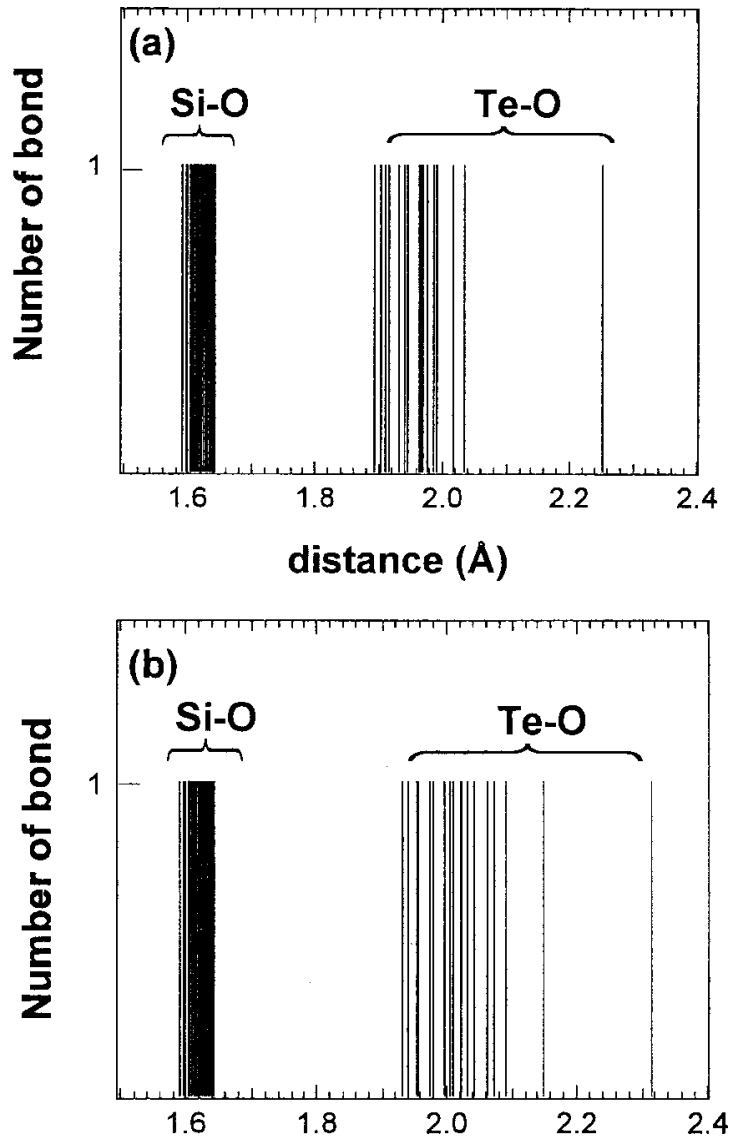

FIG. 8. Te-O bond distances (in $\AA$ ) in the five $\mathrm{TeO}_{4}$ units for model 1 calculated at the (a) HF/3-21G(*) and (b) B3LYP/3-21G(*) levels. Each $\mathrm{Te}-\mathrm{O}$ bond in model 1 corresponds to a vertical line. $\mathrm{Si}-\mathrm{O}$ bond distances in the twelve $\mathrm{SiO}_{4}$ units calculated for the $\mathrm{Si}_{12} \mathrm{O}_{33} \mathrm{H}_{18}$ cluster at the $\mathrm{HF} / 3-21 \mathrm{G}\left({ }^{*}\right)$ level (Ref. 28) are also shown.

of model 2 corresponds to a minimum energy structure from frequency calculations. Figure 3 shows that the basic structure of the respective $\mathrm{Te}_{2} \mathrm{O}_{5}^{2-}$ units in model 3 is similar to that of the isolated $\mathrm{Te}_{2} \mathrm{O}_{5}^{2-}$ (model 2) shown in Fig. 2. It should be noted, however, that some of the nonbridging oxygens in model 3, namely, O7, O16, and O17, interact with the adjacent $\mathrm{Te}$ atoms, forming additional intertrigonal $\mathrm{Te} \cdots \mathrm{O}$ linkages. The distances of the intertrigonal $\mathrm{Te} \cdots \mathrm{O}$ linkages vary from $\sim 2.1$ to $\sim 2.4 \AA$, which are substantially longer than the intratrigonal $\mathrm{Te}-\mathrm{O}$ bond distances in the $\mathrm{TeO}_{3}$ units. It is hence reasonable to assume that these intertrigonal $\mathrm{Te} \cdots \mathrm{O}$ linkages correspond to the longer $\mathrm{Te}-\mathrm{O}$ correlations for $\sim 2.0<r \lesssim 2.4 \AA$ in $\boldsymbol{J}(\boldsymbol{r})\left[\right.$ or $\left.\boldsymbol{J}^{\bmod }(\boldsymbol{r})\right]$ of alkali tellurite glasses shown in Figs. 5 and 6 . In this paper, the terminal oxygens interacting directly with the adjacent $\mathrm{Te}$ atoms are referred to as "virtual bridging oxygen (VBO)" atoms. We also found that the intratrigonal Te-VBO distances in model 2 are comparable to the Te-BO distances, forming three almost equivalent $\mathrm{Te}-\mathrm{O}$ bonds in the respective $\mathrm{TeO}_{3}$ units. We, therefore, suggest that the observed single $\mathrm{Te}-\mathrm{O}$ peak at $\sim 1.9 \AA$ in $\boldsymbol{J}(\boldsymbol{r})$ results from the intertrigonal interactions to form VBO-like atoms in the glass network. 


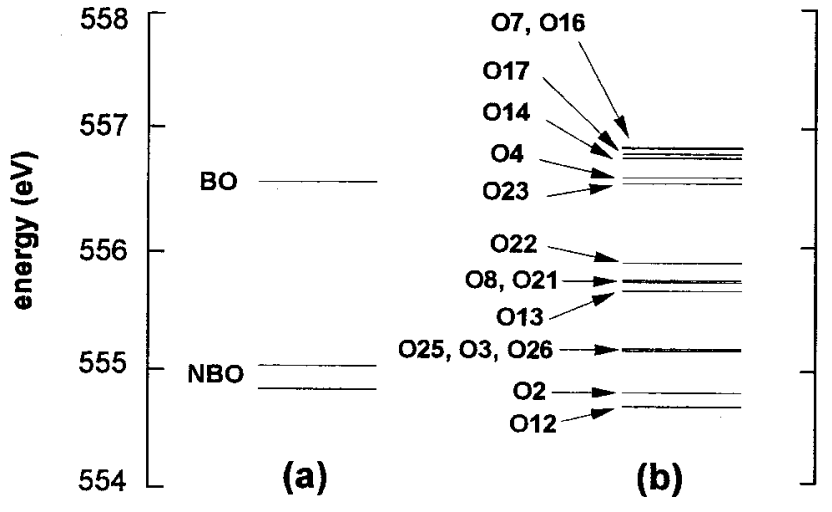

FIG. 9. Calculated $\mathrm{O} 1 s$ photoelectron energies (in eV) of (a) model 2 and (b) model 3 calculated at the HF/3-21G(*) level. For atom labels shown in (b), see Fig. 3.

\section{DISCUSSIONS}

\section{A. $01 s$ photoelectron energies}

Figure 9(a) shows the energy diagram of the O $1 s$ photoelectron energies calculated at the $\mathrm{HF} / 3-21 \mathrm{G}(*)$ level for model 2. It is clear from Fig. 9(a) that the $\mathrm{O} 1 s$ photoelectron energies for the four NBO atoms are basically the same, indicating the resonance between two $\mathrm{Te}-\mathrm{NBO}$ bonds in each $\mathrm{TeO}_{3}$ unit as mentioned above. However, these $\mathrm{O} 1 s$ photoelectron energies of the NBO atoms are substantially lower than that of the $\mathrm{BO}$ atom; the energy difference between the BO and NBO atoms is $\sim 1.5 \mathrm{eV}$. Since the FWHM of the observed O $1 s$ XPS spectra of sodium tellurite glasses is $\sim 1.6 \mathrm{eV},{ }^{14}$ we consider that the above calculated energy difference between the terminal and bridging oxygen atoms is too large and, therefore, model 2 cannot be regarded as a realistic model to represent the electronic structure of sodium tellurite glasses even on the short-range length scale.

Such a discrepancy between the observed and calculated values is most likely due to the neglect of intertrigonal interactions. We then analyze the $\mathrm{O} 1 s$ photoelectron energies of model 3 to investigate how the electronic structure of terminal oxygen atoms is affected by the interaction between the $\mathrm{TeO}_{3}$ units. Figure 9(b) shows the energy diagram of the $\mathrm{O} 1 s$ photoelectron energies calculated for model 3. This diagram can be classified into three energy regions: low$(554.5-555.2 \mathrm{eV})$, mid- $(555.7-556.0 \mathrm{eV})$, and high(556.6-556.9 eV) energy regions.

It has been found that the $\mathrm{O} 1 s$ photoelectron lines in the high-energy region are ascribed not only to the $\mathrm{BO}$ but also to the VBO atoms, namely, O7, O16, and O17. These VBO atoms interact with the adjacent Te atoms to form the intertrigonal linkages as mentioned earlier. The present calculated results hence show that the VBOs yield almost the same $\mathrm{O} 1 s$ photoelectron energies as those of the BOs.

The midenergy region in Fig. 9(b) is composed of four $\mathrm{O} 1 s$ lines. The oxygen atoms that are responsible for this energy region are $08,013, \mathrm{O} 21$, and $\mathrm{O} 22$. On the other hand, the oxygen atoms contributing to the low-energy region are $\mathrm{O} 2, \mathrm{O} 3, \mathrm{O} 12, \mathrm{O} 25$, and $\mathrm{O} 26$. The oxygen atoms contributing to the mid-energy region have intertrigonal $\mathrm{Te}-\mathrm{O}$ interactions in their second coordination shell or in the range from $\sim 3.8$ to $\sim 4.3 \AA$, whereas those contributing to the low-energy region do not have such second-neighbor intertrigonal $\mathrm{Te}-\mathrm{O}$ correlations and hence can be regarded as surface NBO atoms. These calculated results elucidate that when the NBO atoms have first- and second-neighbor Te-O correlations, their $\mathrm{O} 1 s$ photoelectron energies will be increased accordingly. It should also be worth mentioning that the width of the observed $\mathrm{O} 1 \mathrm{~s}$ peak of alkali tellurite glasses is about $1.5-1.7 \mathrm{eV},{ }^{14}$ which is more than the range of midto high-energy regions. Thus, the present $\mathrm{O} 1 s$ photoelectron lines in the mid- along with high-energy regions would show just one band, in agreement with the observed XPS spectra.

On the basis of the present calculated results, we can hence interpret the origin of the single $\mathrm{O} 1 s$ photoelectron line in the XPS spectra of alkali tellurite glasses as follows: (1) Some of the NBO atoms in the glasses can interact with the nearby $\mathrm{Te}$ atoms, forming substantial intertrigonal Te $\cdots$ O linkages, namely, VBO atoms. Consequently, the $1 s$ photoelectron electron energies of the VBOs result in almost the same values as those of the BOs. (2) The rest of the NBO atoms will also interact with Te atoms through the second coordination shell. Such second-neighbor $\mathrm{Te} \cdots \mathrm{O}$ interactions will have an effect to increase their O $1 s$ photoelectron energies as well. In actual alkali tellurite glasses, the NBO atoms will have second-neighbor $\mathrm{Te} \cdots \mathrm{O}$ correlations more than those seen in the present isolated model cluster. Consequently, the energy state of all the NBOs will tend to draw near to that of the $\mathrm{BO}$ atoms, resulting in a single peak in the O $1 s$ XPS spectra of alkali tellurite glasses.

\section{B. Local coordination environments of alkali cations}

We next investigate how the alkali cations interact with the tellurium oxide network on the basis of the cluster calculations. As mentioned before, each $\mathrm{Na}$ atom in model 2 is coordinated by three terminal oxygen atoms. Such a trifurcate coordination of $\mathrm{Na}$ is basically retained in model 3. It should be noted, however, that some of the $\mathrm{Na}$ atoms in model 3 appear to interact with more than three oxygen atoms. In order to get further information about the Na coordination environments, we show in Fig. 10 the distribution of the $\mathrm{Na}-\mathrm{O}$ bond distances calculated for model 3. One sees from Fig. 10 that the first coordination shell of $\mathrm{Na}$ ranges from $\sim 2.1$ to $\sim 2.6 \AA$ for both levels of theory. Furthermore, we have found that the coordination number of sodium cations in their first coordination shell, $N_{\mathrm{Na}}$, changes depending of their cation sites; the values of $N_{\mathrm{Na}}$ vary from 3 to 5 (see Fig. 11). From the measurements of ${ }^{23} \mathrm{Na}$ NMR, the sodium coordination number in tellurite glasses containing over $\sim 30$ mol $\%$ of $\mathrm{Na}_{2} \mathrm{O}$ was estimated to be $\sim 5 .^{29}$ Thus we consider that the local environment of the five-coordinated $\mathrm{Na}$, namely, Na18 in model 3, represents one of the most probable sodium coordination shells in the corresponding actual tellurite glasses.

Figure 11 illustrates the local coordination environment of five-coordinated Na18. The symmetry of this site is far from those of the known symmetric five-coordinate structures, e.g., the trigonal bipyramidal and square pyramidal structures. This indicates that the cation site has a very low symmetry. It should also be worth mentioning that the first 

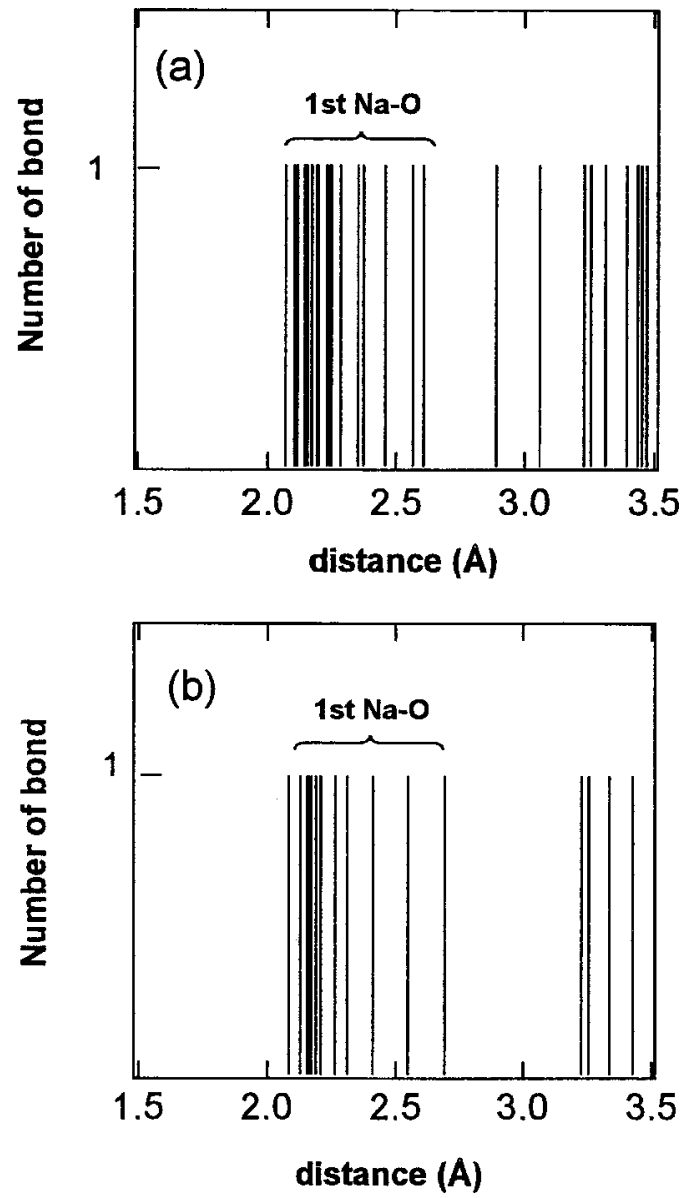

FIG. 10. The distribution of $\mathrm{Na}-\mathrm{O}$ bond distances (in $\AA$ ) calculated for model 3 at the (a) HF/3-21G(*) and (b) B3LYP/3-21G(*) levels. All the bond distances shorter than $3.5 \AA$ are shown as vertical lines.

coordination shell of Na18 is composed of the oxygen atoms that belong to four different $\mathrm{TeO}_{3}$ units in model 3, and this coordination shell is partly shared by other $\mathrm{Na}$ atoms in the same model, e.g., Na9, Na10, Na19. In other words, the $\mathrm{Na}$ atoms may tend to cluster together by partly sharing their first coordination shells. It is hence probable that in actual tellurite glasses the alkali cations will not be homogeneously distributed but will aggregate in the glass network. Recent reverse Monte Carlo modeling has also demonstrated that the sodium cations show significant clustering in tellurite glasses having over $20 \mathrm{~mol} \%$ of $\mathrm{Na}_{2} \mathrm{O} .{ }^{10}$

\section{CONCLUSIONS}

The present radial distribution functions of pure $\mathrm{TeO}_{2}$ as well as alkali tellurite glasses yield several $\mathrm{Te}-\mathrm{O}$ peaks in the first coordination shell of Te. The coordination number of the Te-O peak at $\sim 1.9 \AA$ approaches three with increasing alkali content, indicating the conversion of deformed $\mathrm{TeO}_{4}$ tbps into more regular $\mathrm{TeO}_{3}$ tbs. The longer $\mathrm{Te}-\mathrm{O}$ peaks at $\sim 2.2 \AA$ are probably indicative of the intertrigonal $\mathrm{Te} \cdot \cdots \mathrm{NBO}$ correlations. Our cluster calculations indeed have confirmed that $\mathrm{NBO}$ atoms in the $\mathrm{TeO}_{3}$ unit interact with the adjacent $\mathrm{Te}$ atoms, forming intertrigonal $\mathrm{Te} \cdots \mathrm{NBO}$ linkages in the distance range from $\sim 2.0$ to $\sim 2.4 \AA$. Our cluster calculations have also demonstrated that such intertrigonal

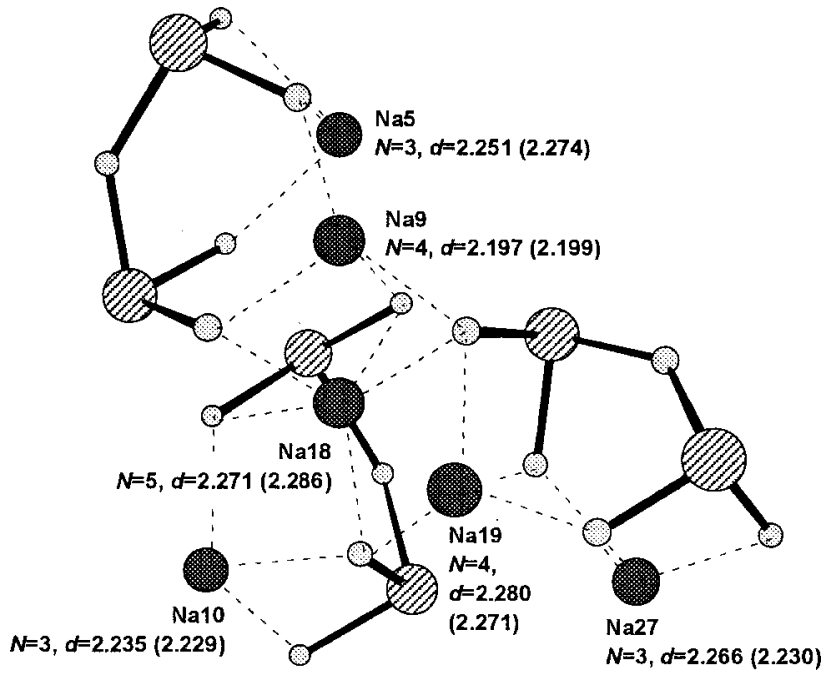

FIG. 11. Local coordination environments of sodium cations in model 3. $\mathrm{Na}-\mathrm{O}$ correlations in their first-coordination shells are indicated by broken lines, corresponding to the coordination number $N$ of each $\mathrm{Na}$ atom. Average $\mathrm{Na}-\mathrm{O}$ bond distances $d$ (in $\AA$ ) for the respective $\mathrm{Na}$ atoms calculated at the $\mathrm{HF} / 3-21 \mathrm{G}\left({ }^{*}\right)$ and $\mathrm{B} 3 \mathrm{LYP} / 3-21 \mathrm{G}\left({ }^{*}\right)$ (values in parentheses) levels are also shown.

$\mathrm{Te} \cdot \mathrm{NBO}$ correlations can also be seen in the secondcoordination shell or in the range from $\sim 3.8$ to $\sim 4.3 \AA$. We have shown that such first- and/or second-neighbor $\mathrm{Te} \cdot \mathrm{NBO}$ interactions have an effect to increase the $1 s$ photoelectron energies of the NBO atoms, which will explain a single peak in the O $1 s$ XPS spectra of alkali tellurite glasses. Thus, we can conclude that in actual alkali tellurite glasses, NBO atoms do not exist in their "pure" form in contrast to the case of conventional oxide glasses.

\section{ACKNOWLEDGMENT}

We would like to thank the Supercomputer Laboratory, Institute for Chemical Research, Kyoto University, for providing the computer time.

${ }^{1}$ J. E. Stanworth, Nature (London) 169, 581 (1952); J. Soc. Glass Technol. 36, 217 (1962).

${ }^{2}$ F. Told, Glastech. Ber. 33, 303 (1960).

${ }^{3}$ R. El-Mallawany, J. Appl. Phys. 72, 1774 (1992).

${ }^{4}$ H. Nasu, O. Matsushita, K. Kamiya et al., J. Non-Cryst. Solids 124, 275 (1990).

${ }^{5}$ S.-H. Kim, T. Yoko, and S. Sakka, J. Am. Ceram. Soc. 76, 2486 (1993).

${ }^{6}$ T. Sekiya, N. Mochida, A. Ohtsuka, and M. Tonokawa, J. Non-Cryst. Solids 144, 128 (1992).

${ }^{7}$ M. Tatsumisago, T. Minami, Y. Kowada, and H. Adachi, Phys. Chem. Glasses 35, 89 (1994)

${ }^{8}$ S. Sakida, S. Hayakawa, and T. Yoko, J. Non-Cryst. Solids 243, 13 (1999).

${ }^{9}$ T. Nishida, S. Saruwatari, and Y. Takashima, Bull. Chem. Soc. Jpn. 61, 4093 (1988)

${ }^{10}$ J. W. Zwanziger, Int. J. Radiat. Phys. Chem. 17, 65 (1998).

${ }^{11}$ J. Kieffer, J. E. Masnik, O. Nickolayev, and J. D. Bass, Phys. Rev. B 58, 694 (1998).

${ }^{12}$ S. R. Nagel, J. Tauc, and B. G. Bagley, Solid State Commun. 20, 245 (1976).

${ }^{13}$ R. Brückner, H.-U. Chun, and H. Goretzki, Glastech. Ber. 51, 1 (1978).

${ }^{14}$ Y. Himei, Y. Miura, T. Nanba, and A. Osaka, J. Non-Cryst. Solids 211, 64 (1997).

${ }^{15}$ T. Uchino, M. Iwasaki, T. Sakka, and Y. Ogata, J. Phys. Chem. 95, 5455 (1991); T. Uchino and T. Yoko, J. Phys. Chem. B 102, 8372 (1998). 
${ }^{16}$ T. Uchino, T. Sakka, Y. Ogata, and M. Iwasaki, J. Phys. Chem. 97, 9642 (1993).

${ }^{17}$ K. Suzuki, M. Misawa, K. Kai, and N. Watanabe, Nucl. Instrum. Methods 147, 519 (1977).

${ }^{18}$ E. Lorch, J. Phys. C 2, 229 (1969).

${ }^{19}$ T. Uchino and T. Yoko, J. Non-Cryst. Solids 204, 243 (1996).

${ }^{20}$ K. D. Dobbs and W. J. Hehre, J. Comput. Chem. 8, 880 (1987), and references therein.

${ }^{21}$ S. Huzinaga, J. Andzelm, M. Klobulowski et al., Gaussian Basis Sets for Molecular Calculations (Elsevier, New York, 1984).

${ }^{22}$ A. D. Becke, J. Chem. Phys. 98, 1372 (1993).
${ }^{23}$ C. Lee, W. Yang, and R. G. Parr, Phys. Rev. B 37, 785 (1988).

${ }^{24}$ T. Koopmans, Physica (Amsterdam) 1, 104 (1933).

${ }^{25}$ R. J. Meier, Chem. Phys. Lett. 138, 471 (1987).

${ }^{26}$ M. J. Frisch, G. W. Trucks, H. B. Schlegel et al., Gaussian 94, Revision D3 (Gaussian, Inc., Pittsburgh, Pennsylvania, 1995).

${ }^{27}$ N. M. Vedishcheva, B. A. Shakhmatkin, M. M. Shultz et al., J. NonCryst. Solids 192\&193, 292 (1995).

${ }^{28}$ T. Uchino and T. Yoko, J. Chem. Phys. 108, 8130 (1998).

${ }^{29}$ S. L. Tagg, R. E. Youngman, and J. W. Zwanziger, J. Phys. Chem. 99, 5111 (1995). 